\title{
Research on Remote Monitoring System of Locomotive Signal Based on 4G Network
}

\author{
Jianhui Zhang ${ }^{\text {a }}$ \\ School of Electrical and Automation Engineering, East China Jiaotong University, Nanchang 330013, \\ China \\ azhang-jh@163.com
}

\begin{abstract}
The railway locomotive signal is a signal installed in the locomotive driver's room to automatically reflect the operating conditions of the front of the train and instruct the driver's operation. It is very important to ensure the safety of the train. The traditional locomotive signal monitoring system has poor real-time performance. In view of this drawback, combining with the mature 4G technology and GPS location technology, a new remote monitoring system of locomotive signal based on $4 \mathrm{G}$ wireless network is presented. The hardware configuration scheme of vehicle terminal is designed, and the software design of ground remote monitoring center is completed. The system can transmit the locomotive signal state information back to the ground monitoring center in real time through the $4 \mathrm{G}$ network, and can realize the remote dynamic monitoring of the locomotive signal and the necessary fault diagnosis.
\end{abstract}

Keywords: remote monitoring, locomotive signal, 4G network.

\section{Introduction}

In recent years, with the rapid development of railway technology, high-speed rail has become a new trend of railway development. From the perspective of transport safety, the requirement for locomotive signal is also increasing. It has gradually changed from the initial repetition signal to the main body signal, and has become an important credential for drivers to control train operation. The subjectivity of locomotive signaling has put forward stricter requirements for its performance, and the corresponding diagnosis and maintenance measures are also facing higher requirements. The traditional diagnosis means of locomotive signal includes on-board inspection on train by railway personnel, track inspection vehicle, and recorder analysis software, etc. Among them, the diagnosis means of on-board inspection on train by railway personnel has lower efficiency, which is time-consuming and laborious, and has great limitations. It can't find random failure factors in time. The diagnosis means of using track inspection vehicle has high monitoring accuracy, but the cost is high, the operation is complex, occupying traffic resources, and it will affect the driving plan and reduce the transportation efficiency. The diagnosis means of using recorder analysis software has defects of the real-time diagnosis is not good enough, and can't detect the possible problems in the train operation in time [1].

Based on this, combined with the current mature 4G technology and GPS positioning technology, the paper presents a solution for remote monitoring system of locomotive signal based on 4G. The system can monitor the operation status of locomotive signal on line, in order to find out the hidden trouble in the train running in time, remotely guide the driver to eliminate the fault, and ensure the traffic safety in real time.

\section{System construction}

With the rapid development of the global wireless communication network, the fourth generation of mobile communication technology (4G) has been quite mature. The 4G technology has obvious advantages in communication speed, transmission quality, and network spectrum. It can access the internet anywhere. It has comprehensive functions of satellite communication, positioning, data 
collection, remote control and so on [2]. Because of this, the presented new remote monitoring system of locomotive signal based on 4G. The system is composed of vehicle terminal, communication network and ground remote monitoring center, as shown in Fig.1 [3]. As an important part of this system, the vehicle terminal is mainly used to collect information about locomotive signal equipment, track circuit signal, locomotive safety information and GPS signal, and send relevant data information to remote monitoring center through $4 \mathrm{G}$ network. The ground remote monitoring center processes and displays the received data, providing operation status of the locomotive signal in real time.

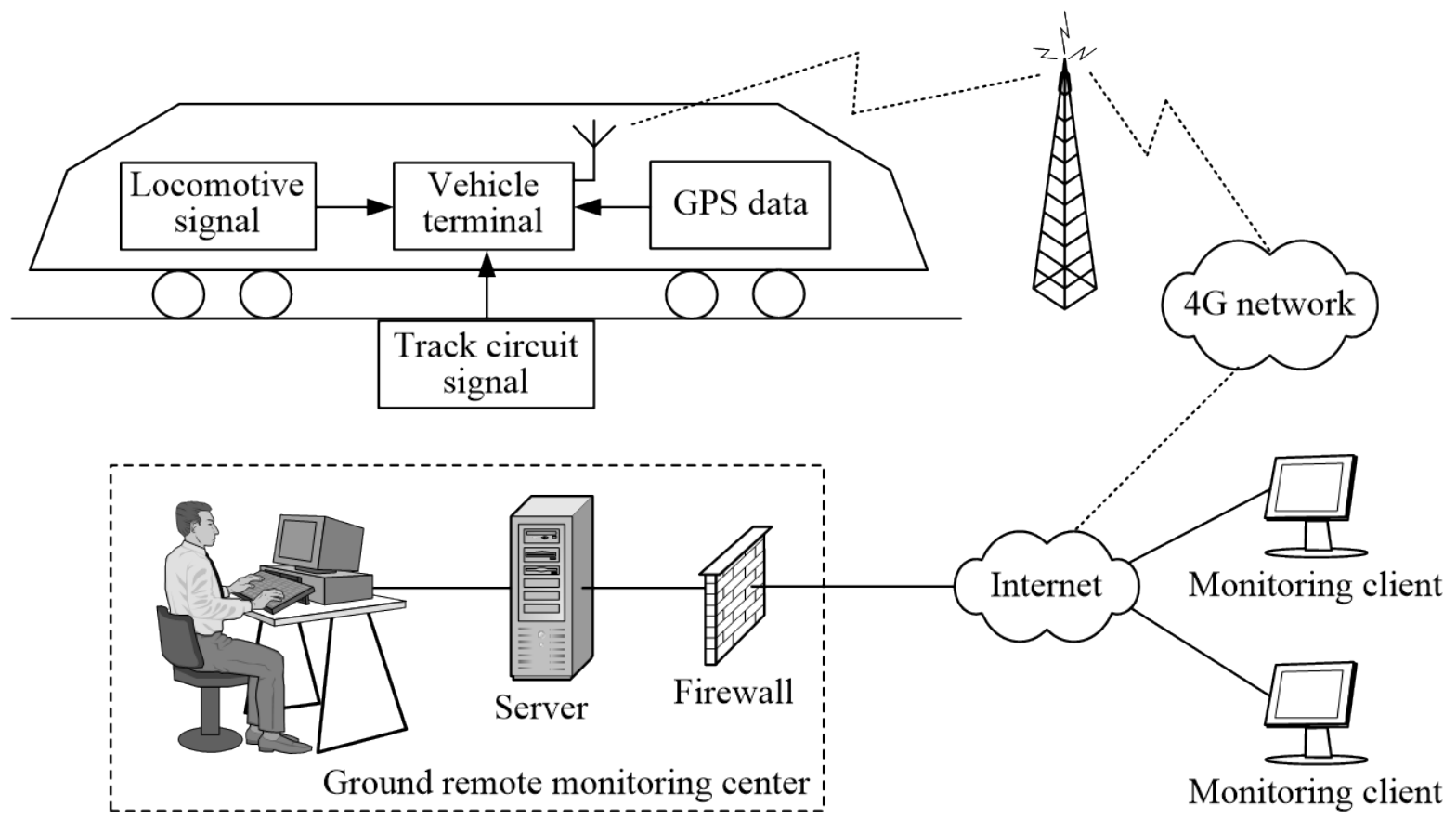

Fig. 1 Diagrammatic sketch of remote monitoring system of locomotive signal

\section{Operational principle of the system}

In the remote monitoring system of locomotive signal showed in Fig.1, the vehicle terminal is an important part of the system. It mainly completes data collection and transmission. It is composed of vehicle terminal host, GPS module and 4G communication module. Among them, the GPS receiver is responsible for receiving the location data of the positioning satellite, and the vehicle terminal host is responsible for extracting relevant data information (which includes the data related to locomotive signal safety and GPS data). It can convert the data into a custom communication protocol format, and send data to the ground monitoring center through 4G network.

The monitoring center adopts a client / server (C/S) mode with high security level and friendly man-machine interface. The server can connect with the vehicle terminal through the $4 \mathrm{G}$ network, receive the information of the locomotive signal status information and train position from the vehicle terminal, and analyze the information and deposit the information into the database. The server mainly completes data analysis, storage and so on, and sends relevant data to the client. The client is mainly human-computer interactive operation. The remote monitoring center stores the received data, and processes the received GPS data, and displays the location information of the train on the monitor screen in real time. The users of the ground monitoring center can query the various information required by the client to access the database, including the locomotive signal state information, the fault information, and the historical data and so on.

\section{Design of the vehicle terminal}

The vehicle terminal installed on the monitored locomotive is the key link to realize remote monitoring for locomotive signal. The information related to locomotive signal safety and location 
information of train must be acquired by the vehicle terminal. The hardware part of vehicle system mainly includes GPS module, 4G module and core processor. The hardware structure of vehicle terminal is shown in Fig.2.

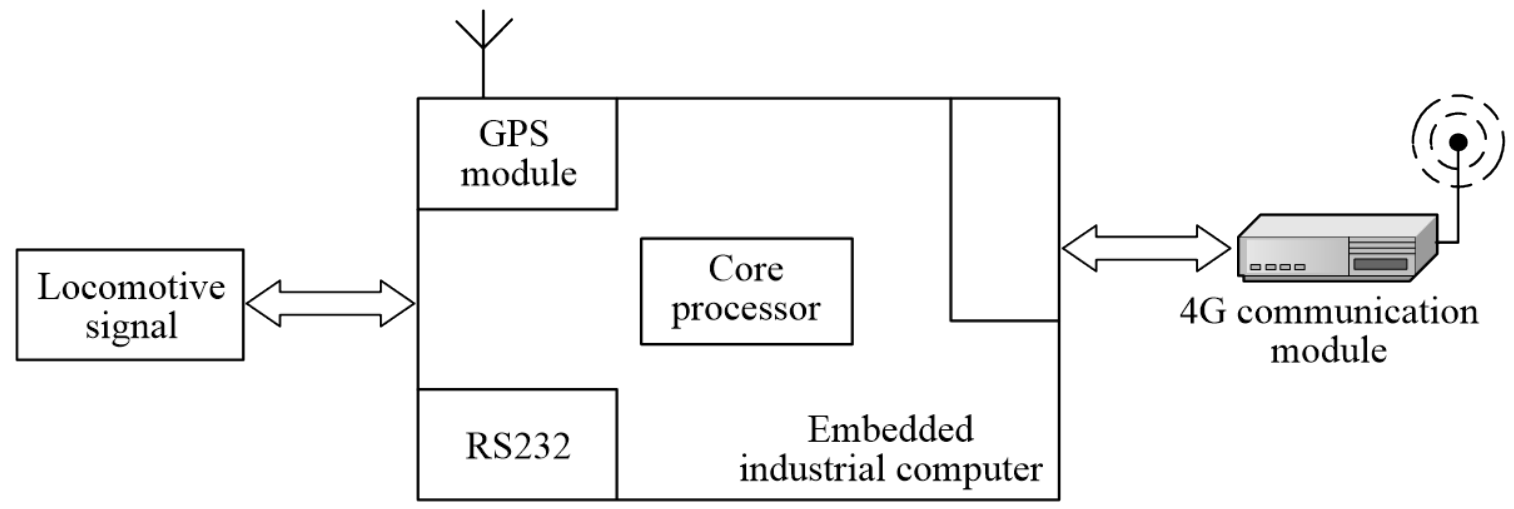

Fig.2 Diagram of vehicle terminal structure

The core processor is the key to realize the function of the vehicle terminal, not only to collect the data of the locomotive signal and the GPS receiver, but also to send the data through the 4G communication module, so it is necessary to have the efficient processing capability. Considering the worse working environment on locomotive, the embedded industrial computer with stable performance is chosen as the terminal host of remote monitoring system for locomotive signal. The GPS module is mainly responsible for receiving the location signal of the GPS satellite, and determines the location of the vehicle terminal based on the location information received. The main function of $4 \mathrm{G}$ communication module is to connect $4 \mathrm{G}$ network, and send data collected from vehicle terminal to ground remote monitoring center through $4 \mathrm{G}$ network.

\section{Design of the system software}

The software part of the system is the soul of the whole system. The software design mainly includes three aspects: vehicle terminal, server side and client. Its schematic diagram is shown as Fig. $3[4]$.

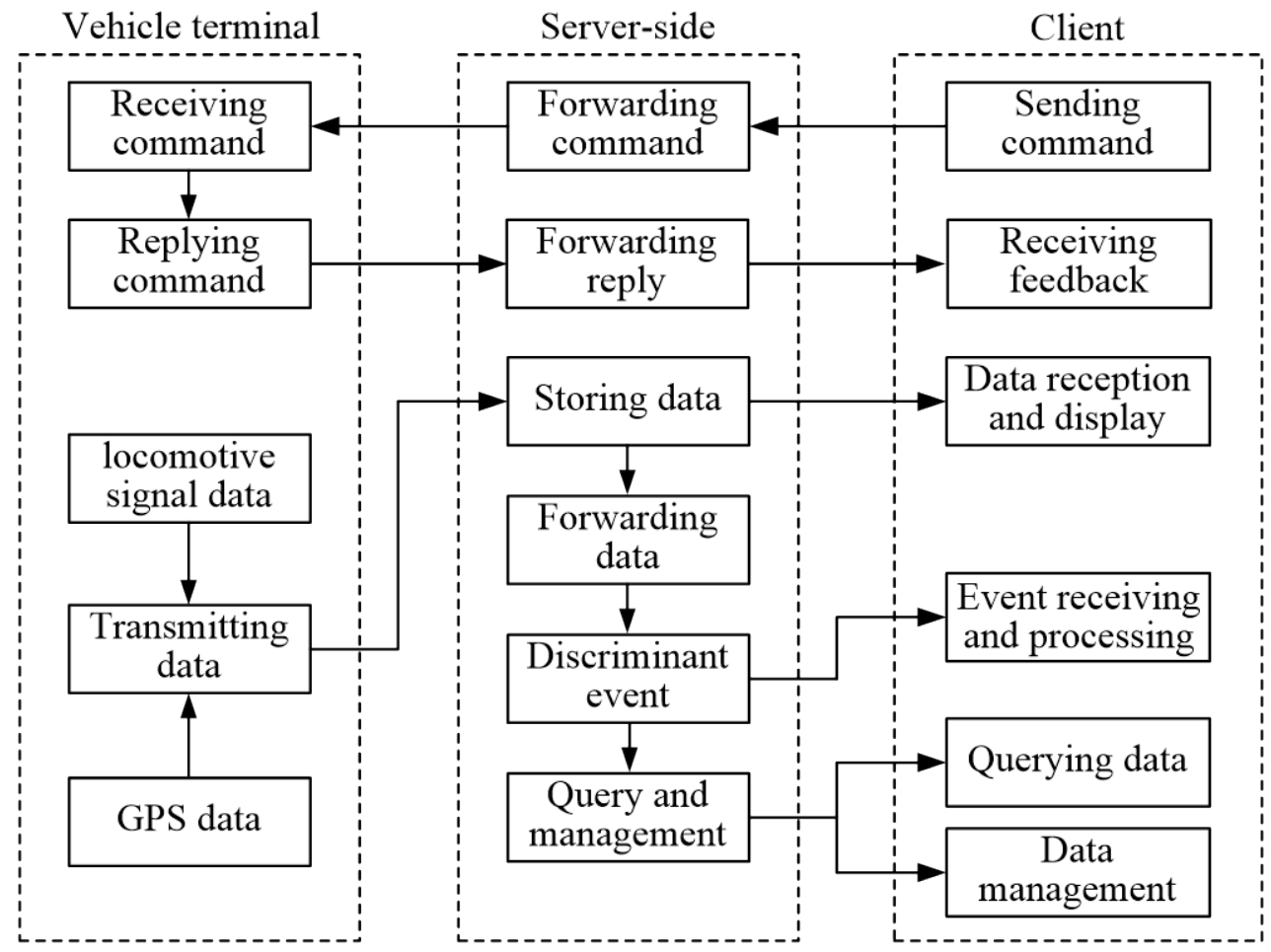

Fig.3 Schematic diagram of system software function 
The vehicle terminal mainly completes data acquisition, including locomotive signal data, GPS data and data transmission. The server side mainly completes data storage and analysis. The client mainly completes the functions of human-computer interaction and other functions. Therefore, the client analysis software provides the human-computer interaction interface of the locomotive signal remote monitoring system. The user can use remote monitoring client software and log on to the remote monitoring server, so the remote monitoring data of locomotive signal can be obtained. The client software represents the remote monitoring data in the form of text and graphics, and the technician is able to diagnose the fault by analyzing the remote monitoring data.

The database is the basis of data storage and operation for remote monitoring system of locomotive signal. The design of database directly affects the monitoring efficiency of remote monitoring system. In the Windows environment, we use the current mainstream database software SQL Server 2016 to develop the database management system. Then create application through Visual $\mathrm{C}++$, and connect to the database for data access. The 'ActiveX Data Objects' in Visual C++ can perform data operations on different data sources, and can quickly create database applications through the common data access objects it provides [5].

\section{Conclusions}

The remote monitoring system of locomotive signal can provide reliable data basis for remote fault diagnosis and accident analysis of locomotive signal. The communication and signal division managers and maintenance personnel can use the remote monitoring system of locomotive signal to analyze the cause of the accident, which can solve the on-site problems in time, so as to effectively ensure the safety of driving. On the other hand, using the remote monitoring system of locomotive signal can effectively improve the management level and maintenance level of locomotive signal equipment, and realize the state repair of the system, which is of great practical significance for improving the comprehensive reliability of the ground and vehicle system.

\section{Acknowledgments}

This work was financially supported by the scientific research project of Jiangxi Provincial Education Department (GJJ160501).

\section{References}

[1] Q.L. Ma, P.X. Du: Railway Signalling \& Communication, Vol. 43 (2007) No.1, p.53.

[2] Information on https://baike.baidu.com/item/4G/523884?fr=aladdin.

[3] Y.Y. Xu: Journal of Yangzhou Polytechnic College, Vol. 18 (2014) No.1, p.50.

[4] H.J. Li: Research on Cab Signal Remote Monitoring System Based on GPRS and GPS (Master, Lanzhou Jiaotong University, China 2014), p.25.

[5] Information on https://blog.csdn.net/foreverhuylee/article/details/35566965. 